\title{
Replacement of NMP solvent for more sustainable, high-capacity, printed Li-ion battery cathodes
}

\author{
Rafal Sliz \\ Optoelectronics and Measurement \\ Techniques Unit \\ University of Oulu \\ 90570 Oulu, Finland \\ rafal.sliz@oulu.fi \\ Tao $\mathrm{Hu}$ \\ Unit of Sustainable Chemistry \\ University of Oulu \\ 90570 Oulu, Finland \\ tao.hu@oulu.fi
}

\author{
Juho Valikangas \\ Unit of Sustainable Chemistry \\ University of Oulu \\ 90570 Oulu, Finland \\ juho.valikangas@oulu.fi
}

\author{
Ulla Lassi \\ Unit of Sustainable Chemistry \\ University of Oulu \\ 90570 Oulu, Finland \\ ulla.lassi@oulu.fi
}

\author{
Pauliina Vilmi \\ Optoelectronics and Measurement \\ Techniques Unit \\ University of Oulu \\ 90570 Oulu, Finland \\ pauliina.vilmi@oulu.fi \\ Tapio Fabritius \\ Optoelectronics and Measurement \\ Techniques Unit \\ University of Oulu \\ 90570 Oulu, Finland \\ tapio.fabritius@oulu.fi
}

\begin{abstract}
The production methods of Li-ion batteries need to be adapted for the goals of a more sustainable future. This research focuses on replacing toxic NMP with less harmful solvents, without compromising the batteries' performance. From the perspective of printed electronics, the replacement is especially desirable because the aggressiveness of NMP has a negative impact on various components of printers. In this research, the novel $\mathrm{NCM88}$ material has been used to fabricate the cathode layers of $\mathrm{Li}$-ion batteries. Two fabrication methods (blade coating and screen printing) and two different slurry/ink formulations (NMP- and DMF-based) have been analysed. For the purpose of physicochemical and electrical characterization, the fabricated cathodes were used to assemble pouch cells and half-cells with metallic lithium as counter electrode. Results indicate that screen-printed cathodes fabricated with DMFbased slurries perform similarly to those fabricated through blade-coating NMP slurries.
\end{abstract}

Keywords: sustainable, DMF, NMP, solvents, battery cathode, NMC, printed, screen printing.

\section{INTRODUCTION}

The rapid development of electronic devices and their demand for portability are creating pressure on developing more effective and longer lasting batteries. Also, recent advances related to electric vehicles and smart grid create additional needs for high-capacity battery systems. Regardless of the application, current battery fabrication methods are not energy efficient or environmentally friendly [1]. According to Ellingsen et al., the fabrication of a $26.6 \mathrm{kWh}$ pack requires energy that is equivalent to approximately 4.6 tonnes of $\mathrm{CO}_{2}$ emitted to the atmosphere [2]. Although technological advancements enable the introduction of more efficient fabrication methods, the ever-increasing demand for batteries poses challenges to meeting sustainability needs [3]. Another aspect is related to toxic and harmful solvents used during battery electrode fabrication. A recent study by Romare and Dahllof concluded that to fabricate a $26.6 \mathrm{kWh}$ battery pack, approximately $23 \mathrm{~kg}$ of N-Methyl-2-Pyrrolidone (NMP) is used [4]. NMP belongs to the group of polar aprotic solvents widely used in the chemical industry. It offers high solvency for several organic and inorganic materials, high boiling point, and thermal stability $[5,6]$. Although up to $99 \%$ of the NMP can be recovered, the recovery process is energy demanding and requires additional investments [7]. Moreover, due to the toxicity of NMP, many countries are introducing legislation that limits its applications [8]. To overcome the problem of NMP usage, several alternatives have been proposed, such as employing water, DMSO, or Cyrene, to name a few [9-11]. Although these efforts provide promising results, they introduce additional challenges related to material solvability, poor adhesion, insertion of sulphur atoms, or delithiation [12], [13].

This work focuses on the replacement of NMP with Dimethylformamide (DMF) and introduces screen printing as a viable fabrication method for battery cathodes (Figure 1). Screen printing is especially promising because it offers controlled material deposition, the flexibility of implementation with a wide range of inks and slurries, the ability to create hybrid devices, and roll-to-roll compatibility [14-16]. Our efforts utilise NMC battery chemistry - LithiumNickel-Manganese-Cobalt-Oxide $\quad\left(\mathrm{LiNiMnCoO}_{2}\right) . \quad \mathrm{NMC}$ batteries offer high specific energy, sufficient lifetime, and high storage capacity. In particular, we utilise a high-capacity novel NMC88 with Ni-Mn-Co ratio of 88-08-03.

Compared to NMP, DMF offers a lower boiling point $\left(153^{\circ} \mathrm{C}\right.$, $\left.\mathrm{NMP}=203{ }^{\circ} \mathrm{C}\right)$, lower surface tension $(37 \mathrm{mN} / \mathrm{m}, \mathrm{NMP}=41$ $\mathrm{mN} / \mathrm{m})$, and lower viscosity $(0.92 \mathrm{mPa} \mathrm{s}, \mathrm{NMP}=1.89 \mathrm{mPa} \mathrm{s})$. All these aspects allow reduction of the energy consumption during electrode drying, improved slurry wetting, and better thermal control of the deposition process $[17,18]$.

Although DMF also belongs to a group of toxic solvents, thanks to the solvent recovery process and its advantageous physicochemical properties, it is a suitable candidate to replace more widely prohibited NMC. The archived results indicate that the DMF-based screen-printed cathodes offer similar performance as their blade-coated NMP-based counterparts.

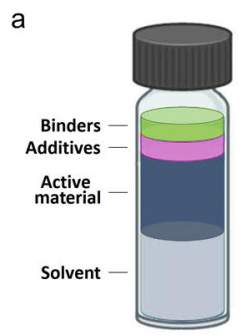

b

Figure 1. a) The NMC slurry composition with approximately $50 \%$ solvent, $46 \%$ active NMC material, $2 \%$ conductive additives, and $2 \%$ binders. b) Screen printing demonstration sketch. 


\section{MethodS}

The NMC slurries were prepared by mixing NMC88 material with conductive carbon black and PVDF binders in a weight ratio of (92:4:4) and concentration of $1 \mathrm{~g} / \mathrm{ml}$. To make a homogenous slurry, two different solvents were used (NMP and DMF) and mixed for $2 \mathrm{~h}$. Accordingly, the NMPbased slurry was used to blade-coat aluminum foil (thickness of $25 \mu \mathrm{m}$ ) serving as current collector, and exposed for drying at $80^{\circ} \mathrm{C}$ for $1 \mathrm{~h}$. The DMF-based slurry was used for screen printing with an Ekra E2 screen printer and Koenen VA 165$0.05 \mathrm{~mm} \mathrm{~W}-\varnothing \times 22.5^{\circ}$ stencils. Consequently, the fabricated films were used for characterisation and battery assembly.

The Scanning Electron Microscopy (SEM) cross-sectional and in-plane images (Figure 2) and Energy-dispersive X-ray spectroscopy (EDS) characterisations of the fabricated NMC films were performed using Zeiss ULTRA plus FESEM Electron Microscope. The elemental analysis (EDS) depicted in Figure 4, was conducted to better understand the material distribution in relation to a fabrication method and solvent dependence. The surface morphology of the fabricated layers depicted in Figure 3 was acquired with Bruker ConturGT optical profilometer in VSI mode.

The fabricated cathodes were used to assemble half-cells (2016-type) with metallic lithium as the counter electrode and $1 \mathrm{M} \mathrm{LiPF} 6$ in EC:DEC:DMC (ratio 1:1:1), as the electrolyte [19]. The half-cells were used to extract information regarding voltage and specific capacity, depicted in Figure 5a.

Pouch cells were assembled using a graphite anode (Hitachi) material and an electrolyte of $1.15 \quad \mathrm{M} \mathrm{LIPF}_{6}$ at EC:DMC:EMC $(2: 4: 4)$ ratio, with the addition of $1 \%$ vinylene carbonate. The pouch batteries were cycled 100 times at a constant charge-discharge current $1 \mathrm{C} / 1 \mathrm{C}$ (Figure $5 b)$.

\section{RESULTS}

The results depicted in Figure 1 indicate a very similar distribution of the NMC88 material, regardless of the used solvent and fabrication method. After the drying process, the average thickness of the NMC layers was 47.8 $\pm 2,1 \mu \mathrm{m}$ and $46.3 \pm 4,4 \mu \mathrm{m}$ for NMP NMC88 Blade-coated and DMF NMC88 Screen-printed, respectively.

The SEM images reveal an interesting difference between samples, related to confinement of the NMC spheres in the layers. The cross-sectional images in Figures $2 \mathrm{a}$ and $2 \mathrm{~b}$ indicate the difference in the surface morphology of the samples. The surface of the DMF NCM88 screen-printed sample is rougher than the surface of the NMP NCM88 bladecoated sample. Figures $2 \mathrm{c}$ and $2 \mathrm{~d}$ demonstrate the distribution of the spheres on the surface of the samples. In the screenprinted sample, the spheres stand out from the surface more significantly than for the blade-coated sample.

The results of optical profilometry characterisation depicted in Figure 3 are in agreement with the previous SEM results, indicating that the DMF NMC88 Screen-printed layer is rougher than the NMP NMC88 Blade-coated layer.

The EDS characterisation of the cross-sectional images presented in Figure 4, shows that the conductive additives and binders are distributed uniformly in the structure of the layers, regardless of the used solvents and fabrication method.
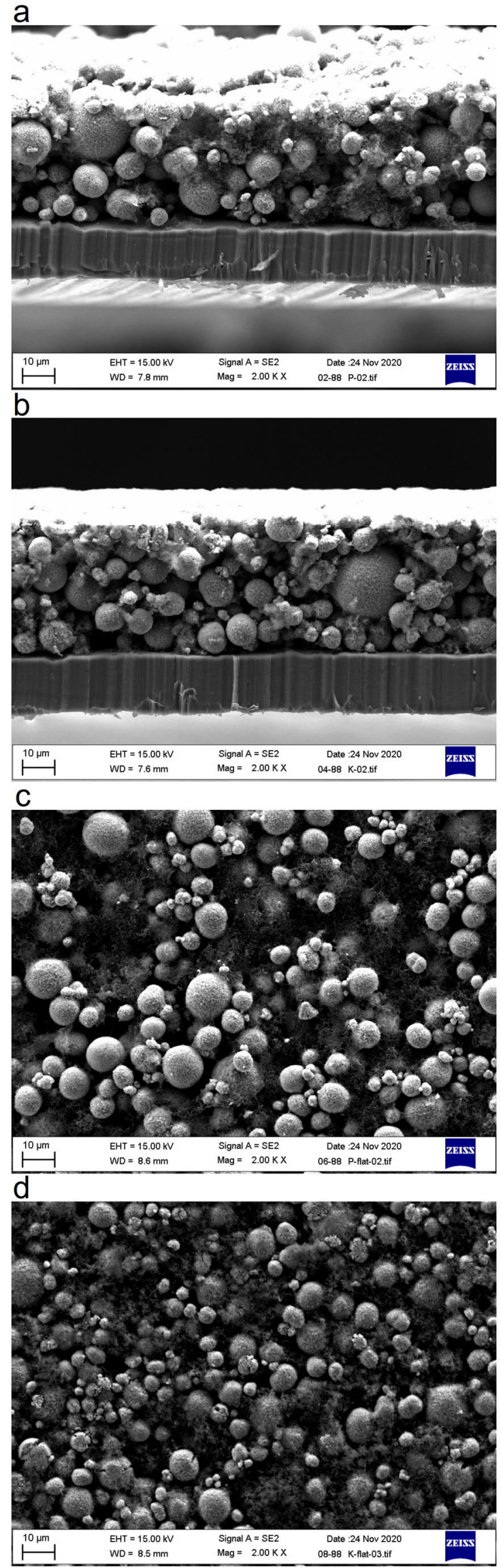

Figure 2. SEM images of the fabricated samples. a) Cross-sectional SEM image of DMF NMC88 Screen-printed. b) Cross-sectional SEM image of NMP NMC88 Blade-coated. c) In-plane SEM image of the DMF NMC88 Screen-printed sample. d) In-plane SEM image of NMP NMC88 Bladecoated sample. 


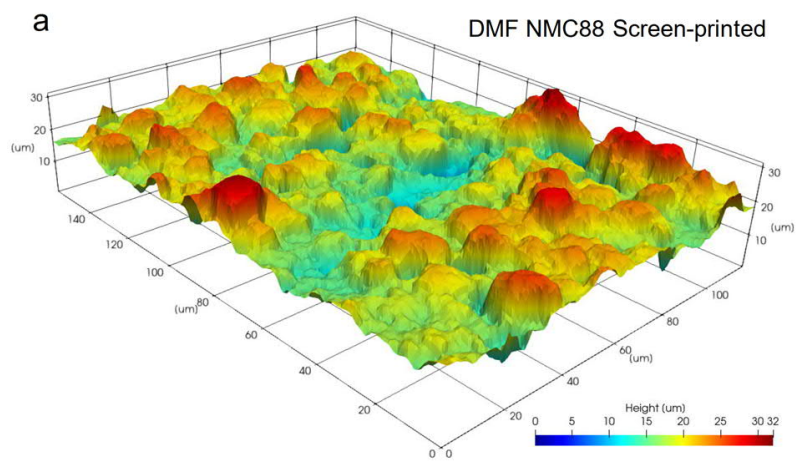

b

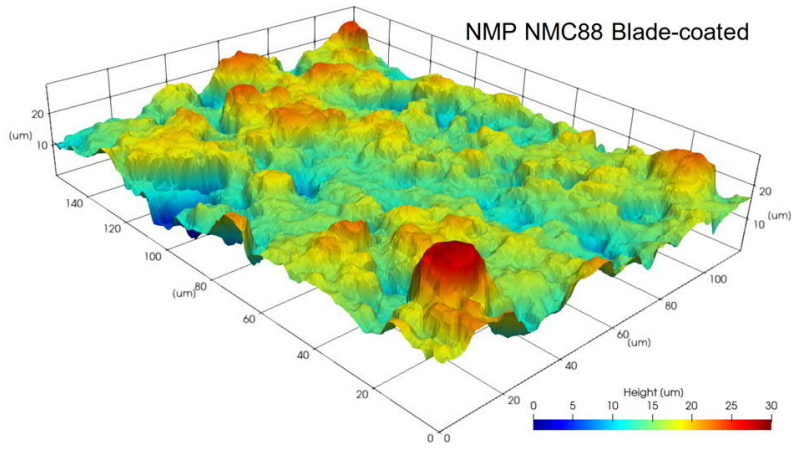

Figure 3. Surface morphology of the fabricated layers acquired with optical profilometry techniques. a) DMF NMC88 Screen-printed layer. b) NMP NMC88 Blade-coated layer.

a
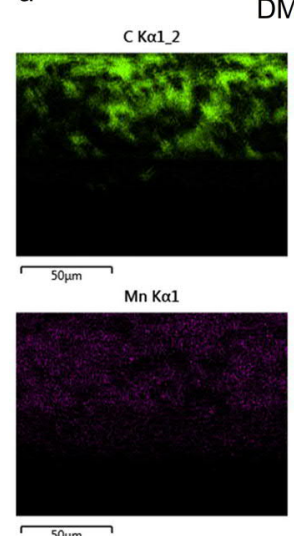

DMF NMC88 Screen-printed
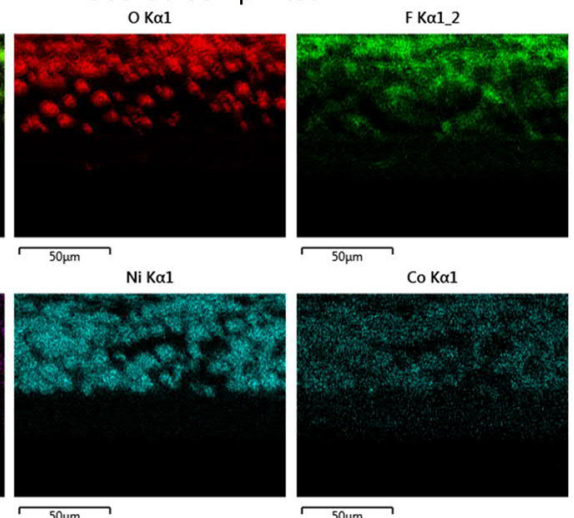

b

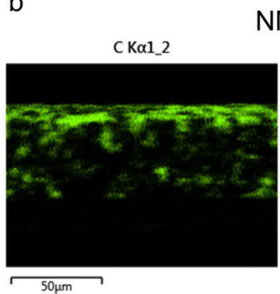

NMP NMC88 Blade-coated
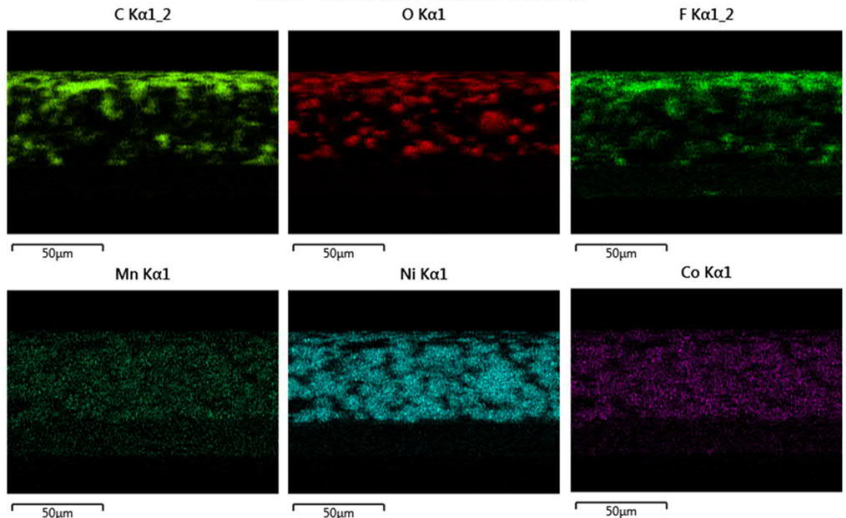

Figure 4. SEM-EDS cross-sectional images of differently fabricated NMC88 layers with an emphasis on elemental distribution of $\mathrm{C}, \mathrm{O}, \mathrm{F}, \mathrm{Mn}, \mathrm{Ni}$, and $\mathrm{Co}$. a) DMF NMC88 Screen-printed layer. b) NMP NMC88 Blade-coated layer.
The electrical analysis depicted in Figure 5 shows the electrical performance of the fabricated batteries. As seen in Figure 5a, both cathodes demonstrate similar charging capacity; however, they reach the plateau at different voltage levels. For the discharge process at various currents, both samples present almost the same behaviour. Figure $5 \mathrm{~b}$ demonstrates the comparison of the cycling process of batteries with differently fabricated cathodes. Regardless of fabrication method, the batteries demonstrate similar capacity fading. The initial values of the specific capacity differ insignificantly in favour of NMP NMC88 Blade-coated samples. However, after x100 cycles, the capacity decreases by $3 \%$ and $2 \%$ for NMP NMC 88 Blade-coated and DMF NMC88 Screen-printed, respectively, favouring the screenprinted sample.

a

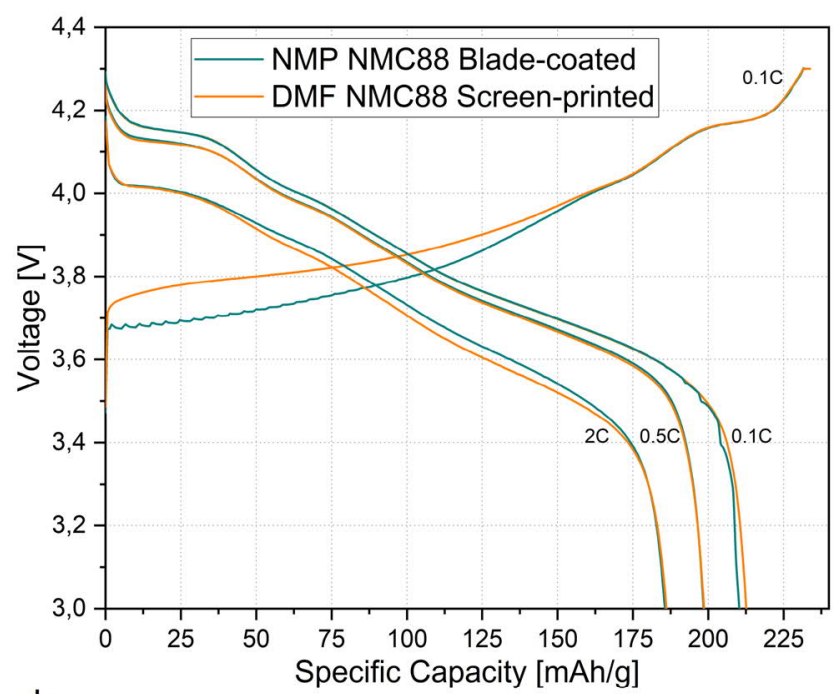

b

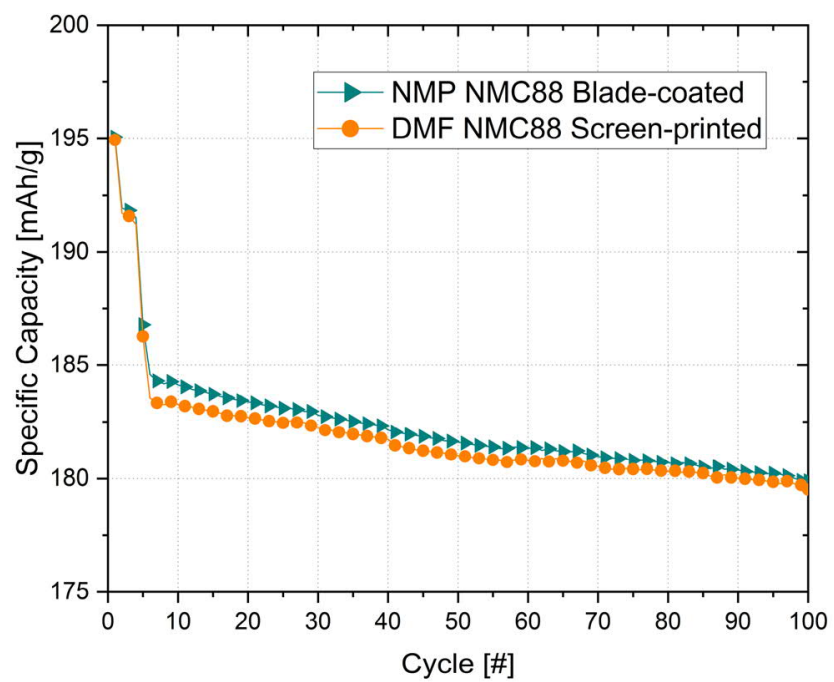

Figure 5. Electrical analysis of the fabricated batteries. a) Specific capacity vs voltage at various charge-discharge current $(0.1 \mathrm{C}, 0.5 \mathrm{C}, 2 \mathrm{C})$ for differently fabricated cathodes. b) Specific capacity during charging-discharging $(1 \mathrm{C} / 1 \mathrm{C})$ process for differently fabricated cathodes.

\section{DISCUSSION AND CONCLUSIONS}

Thanks to the physicochemical and electrical characterisations, this research provides evidence that DMF and screen printing could be viable replacements for toxic NMP and blade-coating. We assume that the morphology 
difference of the layers is associated with the fabrication method. In screen printing, the slurry is pressed through a mesh, while in blade-coating, the blade passes through the surface, allowing better confinement of the NMC spheres. Although the screen-printed layers are rougher, the roughness does not influence the performance of the fabricated batteries. This research could be further advanced by implementing more sophisticated characterisation techniques, such as AFM or electron probe micro analyser, to reduce the deficiencies of optical profilometry or EDS measurements. Furthermore, more cycling and reliability testing would provide additional insights and help in establishing the proposed approach as a viable cathode fabrication method.

The achieved results indicate high potential for the proposed method and ability to replace NMP without compromising the performance of the battery. Thanks to the flexibility and numerous materials compatibility (solvents and active materials), screen printing could be utilised to fabricate various cathode systems and ultimately enable fabrication of fully printed batteries [20].

\section{ACKNOWLEDGMENT}

This research was funded by the EU/Interreg Nord Interegional cooperation project (Project SolBat). The authors acknowledge the help and support of Jessica Nuorala.

\section{REFERENCES}

[1] Y. Liu, R. Zhang, J. Wang, and Y. Wang, "iScience Perspective Current and future lithium-ion battery manufacturing," 2021.

[2] L. A. W. Ellingsen, G. Majeau-Bettez, B. Singh, A. K. Srivastava, L. O. Valøen, and A. H. Strømman, "Life Cycle Assessment of a Lithium-Ion Battery Vehicle Pack," Journal of Industrial Ecology, vol. 18, no. 1, pp. 113-124, Feb. 2014.

[3] E. Emilsson and L. Dahllöf, Lithium-Ion Vehicle Battery Production Status 2019 on Energy Use, CO 2 Emissions, Use of Metals, Products Environmental Footprint, and Recycling. 2019.

[4] M. Romare and L. Dahllöf, The Life Cycle Energy Consumption and Greenhouse Gas Emissions from Lithium-Ion Batteries A Study with Focus on Current Technology and Batteries for light-duty vehicles. 2017.

[5] R. Sliz et al., "Stable colloidal quantum dot inks enable inkjet-printed high-sensitivity infrared photodetectors," ACS Nano, vol. 13, no. 10, 2019.

[6] W. Bauer and D. Nötzel, "Rheological properties and stability of NMP based cathode slurries for lithium ion batteries," Ceramics International, vol. 40, no. 3, pp. 45914598, Apr. 2014.
[7] S. Ahmed, P. A. Nelson, K. G. Gallagher, and D. W. Dees, "Energy impact of cathode drying and solvent recovery during lithium-ion battery manufacturing," Journal of Power Sources, vol. 322, pp. 169-178, Aug. 2016.

[8] J. Sherwood, T. J. Farmer, and J. H. Clark, "Catalyst: Possible Consequences of the N-Methyl Pyrrolidone REACH Restriction," Chem, vol. 4, no. 9. Elsevier Inc, pp. 2010-2012, Sep. 13, 2018.

[9] M. Wang, X. Dong, I. C. Escobar, and Y. T. Cheng, "Lithium Ion Battery Electrodes Made Using Dimethyl Sulfoxide (DMSO) - A Green Solvent," ACS Sustainable Chemistry and Engineering, vol. 8, no. 30, pp. 1104611051, Aug. 2020.

[10] "Can Greener Cyrene Replace NMP for Electrode Preparation of NMC 811 Cathodes?," 2021.

[11] J. Li, Y. Lu, T. Yang, D. Ge, D. L. Wood, and Z. Li, "Water-Based Electrode Manufacturing and Direct Recycling of Lithium-Ion Battery Electrodes-A Green and Sustainable Manufacturing System," iScience, vol. 23, no. 5, p. 101081, May 2020.

[12] R. Sahore, J. Li, and D. L. Wood, "A Study of Factors Responsible for Cracking during Drying of Thick Aqueous-Processed NMC811 Cathodes," ECS Meeting Abstracts, vol. MA2019-02, no. 4, p. 170, Sep. 2019.

[13] G. E. Blomgren, "The Development and Future of Lithium Ion Batteries," Journal of The Electrochemical Society, vol. 164, no. 1, pp. A5019-A5025, Dec. 2017.

[14] R. Sliz et al., "Reliability of R2R-printed, flexible electrodes for e-clothing applications," npj Flexible Electronics, no. 4, 2020.

[15] C. M. Costa, R. Gonçalves, and S. Lanceros-Méndez, "Recent advances and future challenges in printed batteries," Energy Storage Materials, vol. 28, pp. 216-234, Jun. 2020.

[16] Y. Khan, A. Thielens, S. Muin, J. Ting, C. Baumbauer, and A. C. Arias, "A New Frontier of Printed Electronics: Flexible Hybrid Electronics," Advanced Materials, vol. 32, no. 15. Wiley-VCH Verlag, p. 1905279, Apr. 01, 2020.

[17] R. Sliz, Y. Suzuki, T. Fabritius, and R. Myllyla, "Influence of temperature on wetting properties of thin films in organic solar cells applications," Colloids and Surfaces A: Physicochemical and Engineering Aspects, vol. 443, pp. 182-187, 2014.

[18] R. Sliz, Y. Suzuki, A. Nathan, R. Myllyla, and G. Jabbour, "Organic solvent wetting properties of UV and plasma treated $\mathrm{ZnO}$ nanorods: printed electronics approach," Organic Photovoltaics XIII, vol. 8477, p. 84771G, 2012.

[19] J. Välikangas, P. Laine, M. Hietaniemi, T. Hu, P. Tynjälä, and U. Lassi, "Precipitation and Calcination of HighCapacity $\mathrm{LiNiO} 2$ Cathode Material for Lithium-Ion Batteries," Applied Sciences 2020, Vol. 10, Page 8988, vol. 10, no. 24, p. 8988, Dec. 2020.

[20] T. Ma and J. D. MacKenzie, "Fully printed, high energy density flexible zinc-air batteries based on solid polymer electrolytes and a hierarchical catalyst current collector," Flexible and Printed Electronics, vol. 4, no. 1, p. 015010, Mar. 2019. 